\title{
Inhibition of autophagy via activation of PI3K/Akt/mTOR pathway contributes to the protection of hesperidin against myocardial ischemia/reperfusion injury
}

\author{
XUEFEI LI ${ }^{1-3}$, XIAORONG HU ${ }^{1-3}$, JICHUN WANG ${ }^{1-3}$, WEIPAN XU ${ }^{4}$, \\ CHUNFENG $\mathrm{YI}^{5}$, RUISONG MA ${ }^{1-3}$ and HONG JIANG ${ }^{1-3}$ \\ ${ }^{1}$ Department of Cardiology, Renmin Hospital of Wuhan University; ${ }^{2}$ Cardiovascular Research Institute of Wuhan University; \\ ${ }^{3}$ Hubei Key Laboratory of Cardiology, Wuhan, Hubei 430060; ${ }^{4}$ Department of Cardiology, Huangshi Center Hospital, \\ Affiliated Hospital of Hubei Polytechnic University, Huangshi, Hubei 435000; ${ }^{5}$ Department of Cardiology, \\ Wuhan No. 1 Hospital, Wuhan, Hubei 430060, P.R. China
}

Received March 3, 2018; Accepted July 24, 2018

DOI: $10.3892 / \mathrm{ijmm} .2018 .3794$

\begin{abstract}
Hesperidin has been reported to attenuate myocardial ischemia/reperfusion (I/R) injury; however, its effect on autophagy during myocardial $\mathrm{I} / \mathrm{R}$ and the underlying mechanism remains unknown. The present study aimed to investigate whether hesperidin inhibited I/R-induced excessive myocardial autophagy through activating the phosphatidylinositol 3-kinase (PI3K)/protein kinase B (Akt)/mammalian target of rapamycin (mTOR) pathway. Male adult rats were pretreated with hesperidin for a total of 3 days prior to ischemia in the absence or presence of LY294002, a PI3K inhibitor, and then subjected to ischemia for $30 \mathrm{~min}$ followed by reperfusion for $4 \mathrm{~h}$. Myocardial infarct size was measured by Evans blue/triphenyltetrazolium chloride staining. Hematoxylin and eosin staining was used for observing the histological changes in the heart, and the serum levels of creatine kinase-MB (CK-MB) and cardiac troponin I (cTnI) were measured by enzyme-linked immunosorbent assay. Additionally, the protein levels of light chain (LC) 3II, Beclin1, phosphorylated (p)-mTOR, p-Akt and p-PI3K were determined by western blot analysis. Hesperidin pretreatment significantly decreased the myocardial infarct size, myocardial damage and serum levels of CK-MB and cTnI. Furthermore, the expression levels of LC3II and Beclin1 were significantly downregulated and the expression levels of p-mTOR, p-Akt and p-PI3K were markedly upregulated by hesperidin. However, the aforementioned effects as a result of hesperidin were significantly reversed
\end{abstract}

Correspondence to: Dr Hong Jiang, Department of Cardiology, Renmin Hospital of Wuhan University, 238 Jiefang Road, Wuchang, Wuhan, Hubei 430060, P.R. China

E-mail: jianghwurm@163.com

Key words: autophagy, hesperidin, phosphatidylinositol 3-kinase/protein kinase $\mathrm{B} / \mathrm{mammalian}$ target of rapamycin, myocardial ischemia/reperfusion injury, LY294002 by the presence of LY294002. These results demonstrated that hesperidin reduced myocardial I/R injury by suppressing excessive autophagy. Activation of the PI3K/Akt/mTOR pathway contributed to the inhibitory effect of hesperidin on excessive autophagy.

\section{Introduction}

Coronary heart disease, including acute myocardial infarction, is considered to be one of the leading causes of morbidity and mortality worldwide (1). Early and effective reperfusion therapy for acute myocardial infarction can reduce infarct size and improve the clinical outcomes of patients (2). However, reperfusion itself causes ischemia/reperfusion (I/R) injury, which unexpectedly induces additional cellular injury and paradoxically reduces the beneficial effects of reperfusion therapy, leading to irreversible myocardial tissue damage and increased infarct size (2). Therefore, it is necessary to protect against myocardial $I / R$ injury as an adjuvant therapy for the treatment of acute myocardial infarction (3).

Multiple factors such as inflammation, oxidative stress, calcium overload and apoptosis have been identified to contribute to the process of myocardial I/R injury $(2,4,5)$. Autophagy has also been demonstrated to serve an important role in regulating cardiomyocyte death induced by $\mathrm{I} / \mathrm{R}$ (6). Autophagy is a dynamic process that turns over damaged proteins and organelles through a lysosome-associated degradation system and physiologically maintains cellular homeostasis at basal level (7). However, over-activated autophagy induces excessive self-digestion and degradation of essential cellular constituents, which triggers non-apoptotic programmed cell death (8). Emerging evidence has reported that autophagy is upregulated during myocardial $\mathrm{I} / \mathrm{R}$ injury and contributes to cardiomyocyte death; whereas downregulation of autophagy attenuates I/R-induced cardiomyocyte death and protects against myocardial I/R injury (9-11). The results of the aforementioned previous studies indicate that modulation of autophagy may be a target strategy for treatment of myocardial I/R injury. 
Hesperidin [3',5,7-trithydroxy-4'-methoxy-flavanone-7(6- $\alpha$-L-rhamnopyranosyl- $\beta$-D-glucopyranoside)] is a natural flavanone glycoside abundantly present in citrus fruits. It has a molecular formula of $\mathrm{C}_{28} \mathrm{H}_{34} \mathrm{O}_{15}$ and a molecular weight of $610.57 \mathrm{Da}$ (12) (Fig. 1A). Hesperidin possesses good radical scavenging activity and chelating metal ion ability due to its structure, which readily donates electrons from the free hydroxyls on the flavonoid nucleus (13). Furthermore, hesperidin has been reported to exert a wide range of biological effects, including anti-inflammatory (14), anti-cancer (15), radio-protective (16), anti-allergic (17), and antioxidant properties (18). Gandhi et al (19) demonstrated that hesperidin attenuates myocardial I/R injury-induced arrhythmias and apoptosis. Our previous study reported that hesperidin reduced myocardial I/R injury by inhibiting inflammation and oxidative stress (20), however, its effect on autophagy during myocardial I/R and the underlying mechanisms have not been completely elucidated.

The phosphoinositide 3-kinase (PI3K)/protein kinase B (Akt) pathway is known to serve an important role in the control of cell growth, proliferation, survival and metabolism (21). Mammalian target of rapamycin (mTOR), a major intracellular repressor of autophagy, is positively regulated by the PI3K/Akt pathway (22). Our previous study reported that hesperidin could activate the PI3K/Akt pathway (20). Therefore, it was hypothesized that the PI3K/Akt/mTOR pathway may contribute to the regulatory effect of hesperidin on autophagy induced by myocardial I/R injury.

In the present study, the effect of hesperidin on autophagy induced by $\mathrm{I} / \mathrm{R}$ was investigated, and its underlying mechanisms were explored, with particular focus on the potential involvement of the PI3K/Akt/mTOR pathway in the regulation of myocardial autophagy.

\section{Materials and methods}

Animals. Adult male Sprague-Dawley rats (6-8 weeks old; weighing 200-250 g; $\mathrm{n}=56$ ) were supplied by the animal experiment center of Wuhan University (Wuhan, China), and were housed in an air-conditioned room (temperature, $25 \pm 3^{\circ} \mathrm{C}$; humidity, 50-60\%) with free access to food and water on a $12 \mathrm{~h}$ light $/ 12 \mathrm{~h}$ dark cycle. All experimental protocols conformed to the Guideline for the Care and Use of Laboratory Animals published by the US National Institutes of Health and were approved by the Institutional Animal Care and Use Committee of Wuhan University (Approval no. 2016-0318).

Experimental protocol and myocardial I/R model. Hesperidin (HPLC $>98 \%$ ) was purchased from Sinopharm Chemical Reagent Co., Ltd. (Shanghai, China) and dissolved in $0.5 \%$ sodium carboxymethyl-cellulose (CMC-Na) to produce a $100 \mathrm{mg} / \mathrm{ml}$ solution. Then, the solution of hesperidin or $0.5 \%$ CMC-Na was administered orally to rats by gavage for a total of 3 days $(20,23)$. After being anesthetized with an intraperitoneal injection of sodium pentobarbital $(45 \mathrm{mg} / \mathrm{kg}$; Sigma-Aldrich; Merck KGaA, Darmstadt, Germany), all animals were artificially ventilated using a volume-controlled rodent respirator and monitored with an electrocardiogram using a computer-based EP system (LEAD2000B; Jinjiang Ltd., Chengdu, China). A thoracotomy through a left parasternal
A
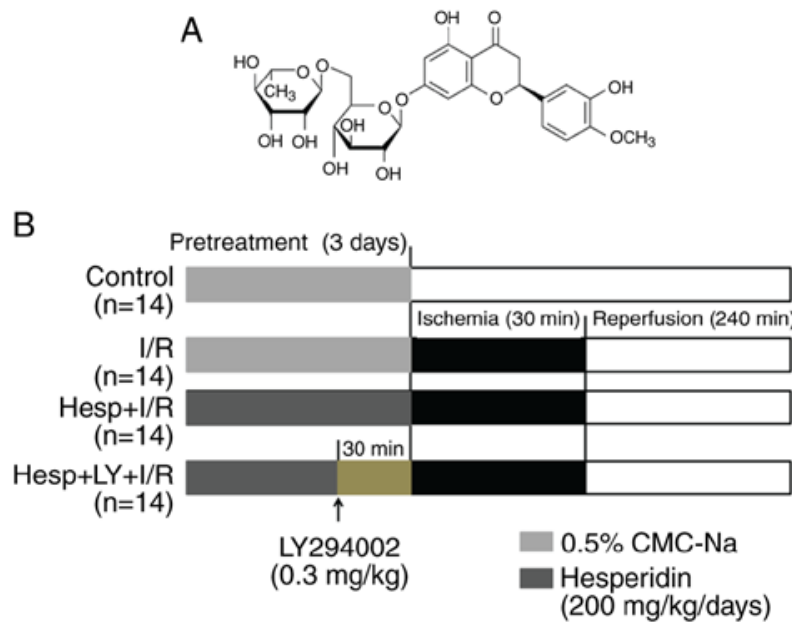

Figure 1. (A) The structure of hesperidin and (B) The study protocol for animal groups subjected to $30 \mathrm{~min}$ ischemia following $240 \mathrm{~min}$ reperfusion. CMC-Na, sodium carboxymethyl-cellulose; Hesp, hesperidin; I/R, ischemia/reperfusion; LY, LY294002.

incision was performed to expose the heart. A 4-0 silk was passed under the left anterior descending coronary artery (LAD). The myocardial I/R model was induced by ligation and release of the silk. A successful myocardial I/R model was verified by changes of ST segment elevation in Lead-II and regional cyanosis of the myocardial surface.

A total of 56 rats were randomly divided into the following four groups ( $n=14$ per group): i) Sham operated control (Control): $0.5 \%$ CMC-Na; ii) Ischemia/reperfusion (I/R): 0.5\% CMC-Na; iii) Hesperidin ( $200 \mathrm{mg} / \mathrm{kg} /$ day $)+\mathrm{I} / \mathrm{R}$ (Hesp+I/R); or iv) Hesperidin (200 mg/kg/day) + LY294002 $+\mathrm{I} / \mathrm{R}(\mathrm{Hesp}+\mathrm{LY}+\mathrm{I} / \mathrm{R})$ : Following a 3 day pretreatment with hesperidin $(200 \mathrm{mg} / \mathrm{kg} / \mathrm{day})(20)$, rats in the Hesp+LY+I/R group were administered LY294002 [a specific PI3K inhibitor, $0.3 \mathrm{mg} / \mathrm{kg}$ (24), Sigma-Aldrich; Merck KGaA] via a caudal vein 30 min prior to LAD occlusion. Rats in the Control group were subjected to surgical manipulation without ligaturing the LAD; however, rats in the remaining groups were subjected to $30 \mathrm{~min}$ of LAD occlusion followed by a $4 \mathrm{~h}$ reperfusion. At the end of the experiment, the rats were sacrificed, and their blood samples and hearts were harvested for subsequent analysis. The study protocol is illustrated in Fig. 1B.

Myocardial infarct size. Myocardial infarct size was assessed by 2,3,5-triphenyltetrazolium chloride (TTC; Sigma-Aldrich; Merck KGaA) as previously described $(19,25)$. Briefly, following reperfusion, the LAD was occluded again and $2 \mathrm{ml}$ of $1 \%$ Evans blue dye (Sigma-Aldrich; Merck KGaA) was infused via the femoral vein. Hearts were removed and cut $(\sim 2 \mathrm{~mm})$ from apex to base. The slices were incubated in $1 \%$ TTC at $37^{\circ} \mathrm{C}$ for $20 \mathrm{~min}$ and fixed in $4 \%$ paraformaldehyde at room temperature overnight. The infarct area (white) and the risk area (red) in each section were determined by Image-Pro Plus 6.0 (Media Cybernetics, Inc., Rockville, MD, USA). A total of 6 animals in each group were used for myocardial infarct size measurement. Myocardial infarct size was expressed as a percentage of the risk area volume [\%, infarct area / (risk + infarct area)]. 
Myocardial injury. Serum levels of creatine kinase-MB (CK-MB) and cardiac troponin I (cTnI) were used as indicators of myocardial injury. Blood samples were centrifuged at $1,000 \mathrm{x} \mathrm{g}$ for $15 \mathrm{~min}$ at room temperature, and the serum was collected for analysis. Standard techniques were performed to determine the serum levels of CK-MB and cTnI by using commercial kits (cat. no. E006; Nanjing Jiancheng Bioengineering Institute, Nanjing, China) and enzyme-linked immunosorbent assay kits (cat. no. E-EL-R0055c; Elabscience, Wuhan, China), each procedure was conducted according to the manufacturer's protocol.

Western blot analysis. Total protein extracts from the rat heart tissue were prepared as previously described (26). Protein extraction was performed using a radioimmunoprecipitation assay lysis buffer (cat. no. P0013B; Beyotime Institute of Biotechnology, Jiangsu, China), and the concentration of the protein was measured by a bicinchoninic acid protein assay kit (cat. no. P0010; Beyotime Institute of Biotechnology). A total of $50 \mu \mathrm{g}$ protein per lane was electrophoresed on $10 \%$ sodium dodecyl sulfate-polyacrylamide gel and transferred to a nitrocellulose membrane. The membranes were blocked with 5\% non-fat dry milk in Tris-buffer saline- $0.05 \%$ Tween for $2 \mathrm{~h}$ at room temperature and incubated respectively at $4^{\circ} \mathrm{C}$ overnight with the following primary antibodies including: Anti-light chain (LC)3 (cat. no. 2775; 1:1,000), Beclin1 (cat. no. 3738; 1:1,000), mTOR (cat. no. 2972S; 1:1,000), phosphorylated (p)-mTOR (cat. no. 2971S; 1:1,000), Akt (cat. no. 4691; 1:1,000), p-Akt (cat. no. 4060; 1:2,000), p-PI3K (cat. no. 4228; 1:1,000) and PI3K (cat. no. 4257; 1:800) (Cell Signaling Technology, Inc., Danvers, MA, USA). The membrane was then washed and incubated with a horseradish peroxidase conjugated secondary antibody (cat. no. BA1054; 1:50,000, Wuhan Boster Biological Technology, Ltd., Wuhan, China) at $37^{\circ} \mathrm{C}$ for $2 \mathrm{~h}$. The protein bands were visualized by an enhanced chemiluminescence system (Thermo Fisher Scientific, Inc., Waltham, MA, USA), and glyceraldehydes-3-phosphate dehydrogenase (GAPDH; cat. no. BM3896; 1:400; Wuhan Boster Biological Technology, Ltd.) was used as an internal control to correct the variation between samples. The densitometry of each band was quantified by Image-Pro Plus 6.0 (Media Cybernetics, Inc.). A total of 5 hearts in each group were used for the western blot analysis.

Immunofluorescence staining. To observe the LC3 expression in the myocardial tissue, immunofluorescence staining was performed as described previously (27). Following deparaffinization, the paraffin-embedded sections $(5 \mu \mathrm{m})$ were replaced in the citrate buffer $(\mathrm{pH}$ 6.0) and boiled with microwave on full power for antigen retrieval. After cooling, the sections were washed with PBS ( $\mathrm{pH} 7.4$ ) three times ( 3 min each), and blocked with 3\% goat serum (cat. no. AR1009; Wuhan Boster Biological Technology, Ltd.) for $30 \mathrm{~min}$ at room temperature. The sections were then incubated with anti-LC3 antibody (1:100; Cell Signaling Technology, Inc.) at $4^{\circ} \mathrm{C}$ overnight. Then, the sections were incubated with a FITC conjugated secondary antibody (cat. no. BA1105; 1:40; Wuhan Boster Biological Technology, Ltd.) at $37^{\circ} \mathrm{C}$ for $1 \mathrm{~h}$. Nuclei were stained with 4',6-diamidino-2-phenylindole (DAPI; Sigma-Aldrich; Merck KGaA) for $5 \mathrm{~min}$ at room temperature in the dark. A total of 6 fields at x200 magnification were randomly selected from 3 sections in each group and visualized with a fluorescence microscope (excitation wavelength: 495 nm; DX51; Olympus Corporation, Tokyo, Japan). The mean densitometry of the fluorescence signal of LC3 in each field was analyzed by Image-pro plus 6.0 software (Media Cybernetics, Inc.).

Hematoxylin and Eosin staining. Myocardial tissue was fixed in $4 \%$ paraformaldehyde, routinely processed and embedded in paraffin. Paraffin-embedded sections were stained with hematoxylin for $7 \mathrm{~min}$, and eosin for $15 \mathrm{sec}$ at room temperature. A total of 6 fields at x 200 magnification were randomly selected from 3 sections in each group and visualized with a light microscope to observe the myocardium damage.

Statistical analysis. All continuous data are expressed as the mean \pm standard deviation and analyzed using SPSS software, version 19.0 (IBM SPSS, Armonk, NY, USA). A one-way analysis of variance was used for comparisons among groups with Bonferroni's adjustment for multiple comparisons. Each experiment was repeated 3 times. All plotting graphics were created using the GraphPad Prism 6.0 software (GraphPad Software, Inc., La Jolla, CA, USA). P $<0.05$ was considered to indicate a statistically significant difference.

\section{Results}

Hesperidin decreases myocardial infarct size during $I / R$. To investigate the cardioprotective effects of hesperidin against $\mathrm{I} / \mathrm{R}$, the myocardial infarct size was determined. As presented in Fig. 2, when compared with the I/R group, the Hesp+I/R group exhibited a marked decrease in the myocardial infarct size. However, the addition of LY294002 partially inhibited the cardioprotective effect of hesperidin $(\mathrm{P}<0.05)$.

Hesperidin alleviates cardiac injury induced by $I / R$. The damage of myocardium induced by $\mathrm{I} / \mathrm{R}$ was further confirmed by hematoxylin and eosin staining and the serum levels of CK-MB and cTnI. As presented in Fig. 3A, the myocardial structure in the I/R group exhibited ruptured cardiac muscle fibers, necrosis with inflammatory cell infiltration and interstitium edema; however, no such damage was observed in the Control group. Hesperidin treatment prevented myocardial damage after $\mathrm{I} / \mathrm{R}$, and the myocardium exhibited rescued fiber disruption, necrosis and inflammatory cell infiltration. However, the administration of LY294002 abolished these effects. The serum CK-MB and cTnI levels were also determined to assess myocardial injury (Fig. 3B and C). Compared with the Control group, the serum CK-MB and cTnI levels were significantly increased in the $\mathrm{I} / \mathrm{R}$ group $(\mathrm{P}<0.05)$. The serum CK-MB and cTnI levels were significantly lower in the Hesp+I/R group compared with the I/R group. However, LY294002 partially reversed these effects $(\mathrm{P}<0.05)$.

Hesperidin decreases autophagy in hearts subjected to $I / R$. The present study first detected the regulatory effect of hesperidin on LC3 and Beclin1 using immunofluorescence staining and western blot analysis. As presented in Fig. 4A, the LC3 (green) signals increased in the I/R group when 
A
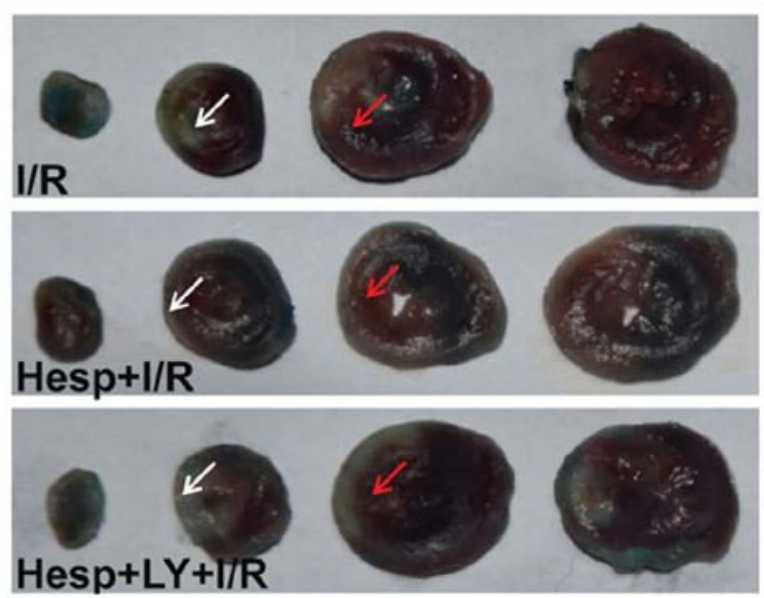

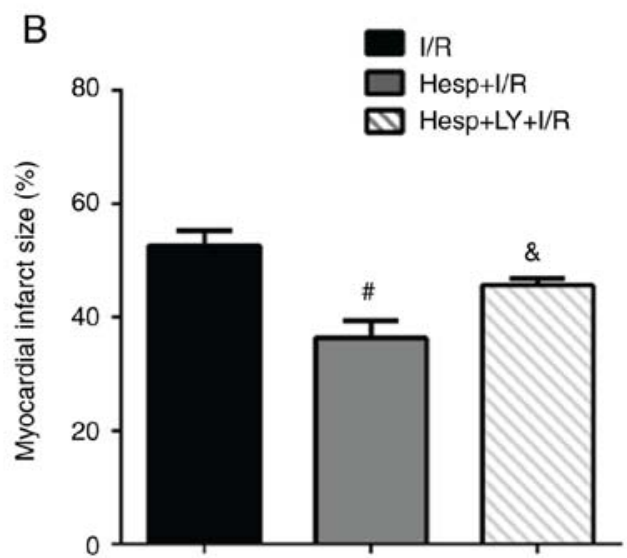

Figure 2. Effect of hesperidin on myocardial infarct size after I/R. (A) Representative images of 2,3,5-triphenyltetrazolium chloride stained samples showing the infarct area (white arrows) and risk area (red arrows). (B) Myocardial infarct size (n=6). ${ }^{\text {P }}<0.05$ vs. I/R group; ${ }^{\star} \mathrm{P}<0.05$ vs. Hesp+I/R group. Hesp, hesperidin; I/R, ischemia/reperfusion; LY, LY294002.
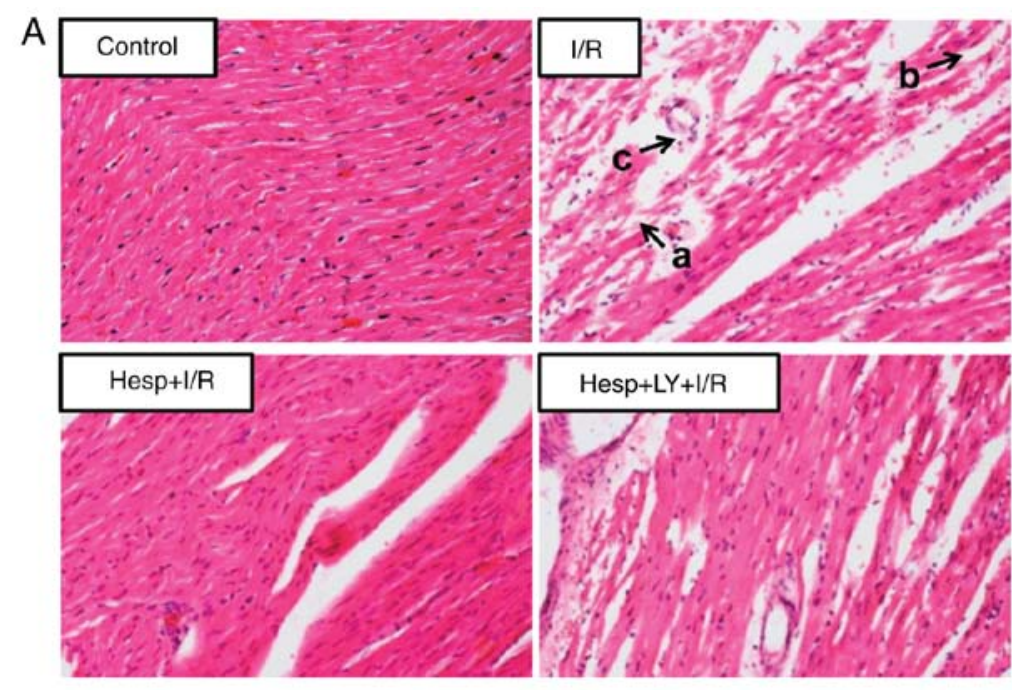

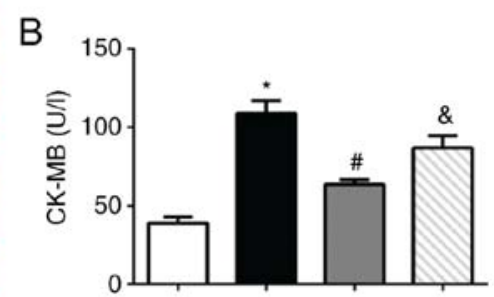

C

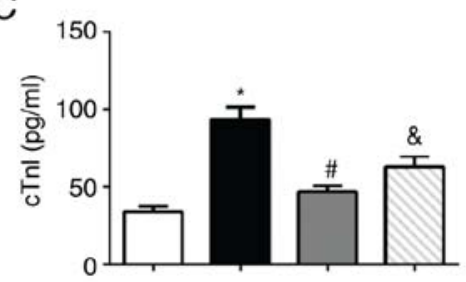

Control

- I/R

$\square$ Hesp+l/R

$\square \mathrm{Hesp}+\mathrm{LY}+\mathrm{l} / \mathrm{R}$

Figure 3. Effect of hesperidin on cardiac injury induced by I/R. (A) Representative images from hematoxylin and eosin staining (x200 magnification) indicating histopathological changes in the myocardium: a, ruptured myocardial fiber; b, edema in between myocardial fibers; $c$, recruited inflammatory cells. (B) Serum level of CK-MB (n=6). (C) Serum level of $\mathrm{cTnI}(\mathrm{n}=6)$. ${ }^{\mathrm{P}} \mathrm{P}<0.05$ vs. Control group; ${ }^{~} \mathrm{P}<0.05$ vs. I/R group; ${ }^{\mathrm{P}} \mathrm{P}<0.05$ vs. Hesp+I/R group. CK-MB, creatine kinase-MB; cTnI, cardiac troponin I; Hesp, hesperidin; I/R, ischemia/reperfusion; LY, LY294002.

compared with the Control group. Hesperidin substantially decreased the I/R-induced LC3 signal accumulation; however, its effect was abrogated by LY294002. The mean densitometry values of LC3 and expression levels of LC3II and Beclin1 were significantly increased in the I/R group when compared with those in the Control group $(\mathrm{P}<0.05$; Figs. $4 \mathrm{~B}$ and 5$)$. Hesperidin markedly suppressed the increased mean densitometry values of LC3 and expression levels of LC3II and Beclin1 induced by $\mathrm{I} / \mathrm{R}(\mathrm{P}<0.05)$, which indicated that hesperidin could inhibit I/R-induced autophagy. However, administration of LY294002 partially abolished these effects $(\mathrm{P}<0.05)$.

Hesperidin increases expression levels of $p$-mTOR, $p$-Akt and $p$-PI3K during myocardial I/R. To investigate the possible mechanism underlying the inhibitory effect of hesperidin on autophagy during myocardial I/R injury, the expression levels of p-mTOR, mTOR, p-Akt, Akt, p-PI3K and PI3K in the myocardium were examined using western blot analysis. As presented in Fig. 6, compared with the Control group, the p-mTOR, p-Akt and p-PI3K expression levels were significantly decreased in the $\mathrm{I} / \mathrm{R}$ group $(\mathrm{P}<0.05)$. Hesperidin treatment markedly inhibited the decrease in the p-mTOR, $\mathrm{p}$-Akt and p-PI3K expression levels, demonstrating that hesperidin could activate the $\mathrm{PI} 3 \mathrm{~K} / \mathrm{Akt} / \mathrm{mTOR}$ pathway; however, LY294002 significantly reversed these effects $(\mathrm{P}<0.05)$. Additionally, no significant difference in mTOR, Akt and PI3K expression levels were observed among the four groups $(\mathrm{P}>0.05)$. 
A
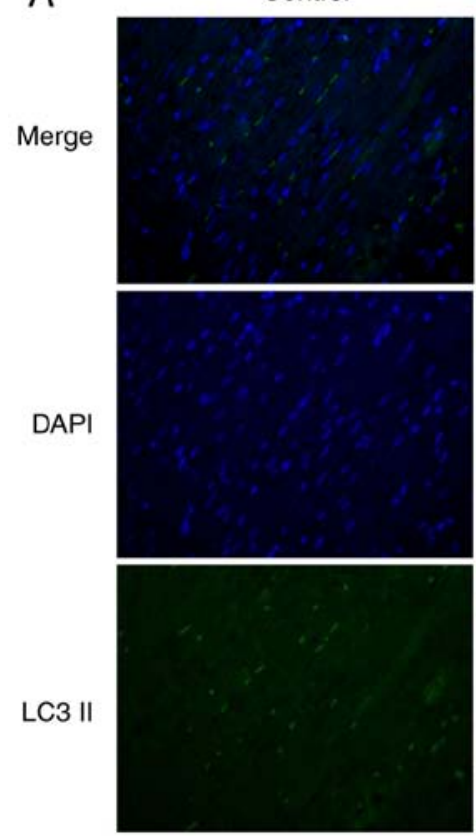

B

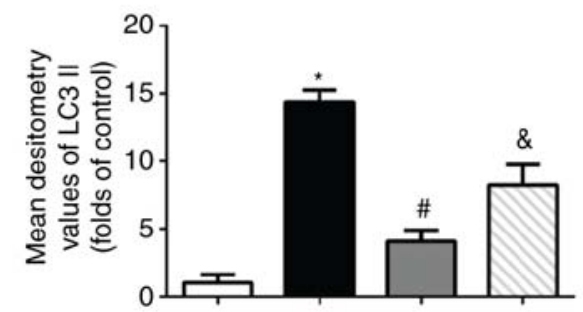

I/R
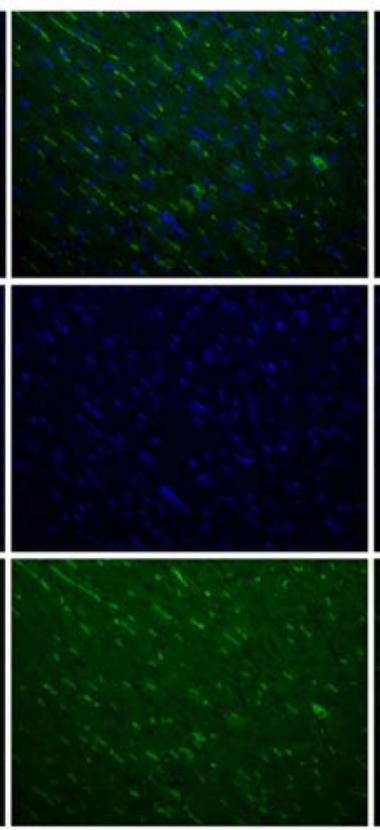

Hesp+l/R
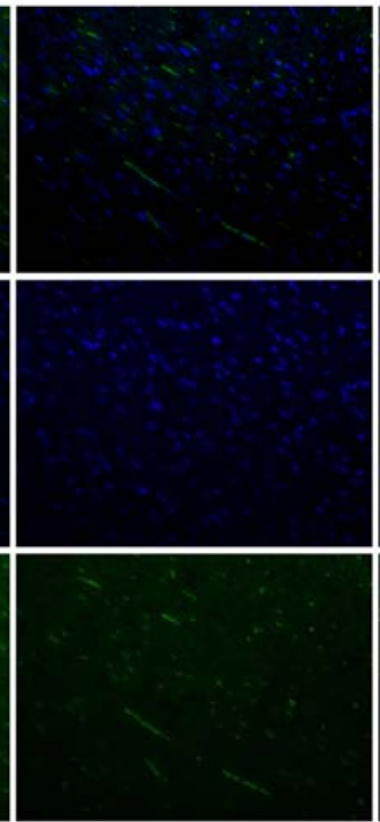

$\mathrm{Hesp}+\mathrm{LY}+\mathrm{l} / \mathrm{R}$
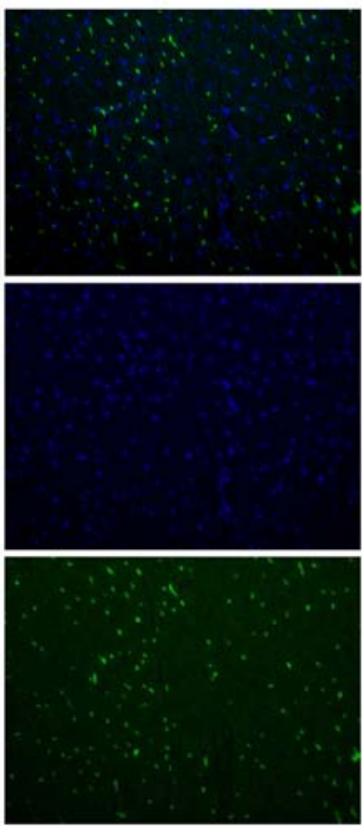

Figure 4. Effect of hesperidin on LC3 II expression level in the heart subjected to I/R. (A) Representative images of immunofluorescence staining (x200 magnification) demonstrating the expression of LC3 II (green) in the myocardium. (B) Fold changes of the mean densitometry values of LC3 II ( $\mathrm{n}=6$ ). ${ }^{*} \mathrm{P}<0.05$ vs. Control group; ${ }^{\text {P }}<0.05$ vs. I/R group; ${ }^{\circledR} \mathrm{P}<0.05$ vs. Hesp+I/R group. DAPI, 4',6-diamidino-2-phenylindole; Hesp, hesperidin; I/R, ischemia/reperfusion; LY, LY294002; LC3 II, light chain 3 II.
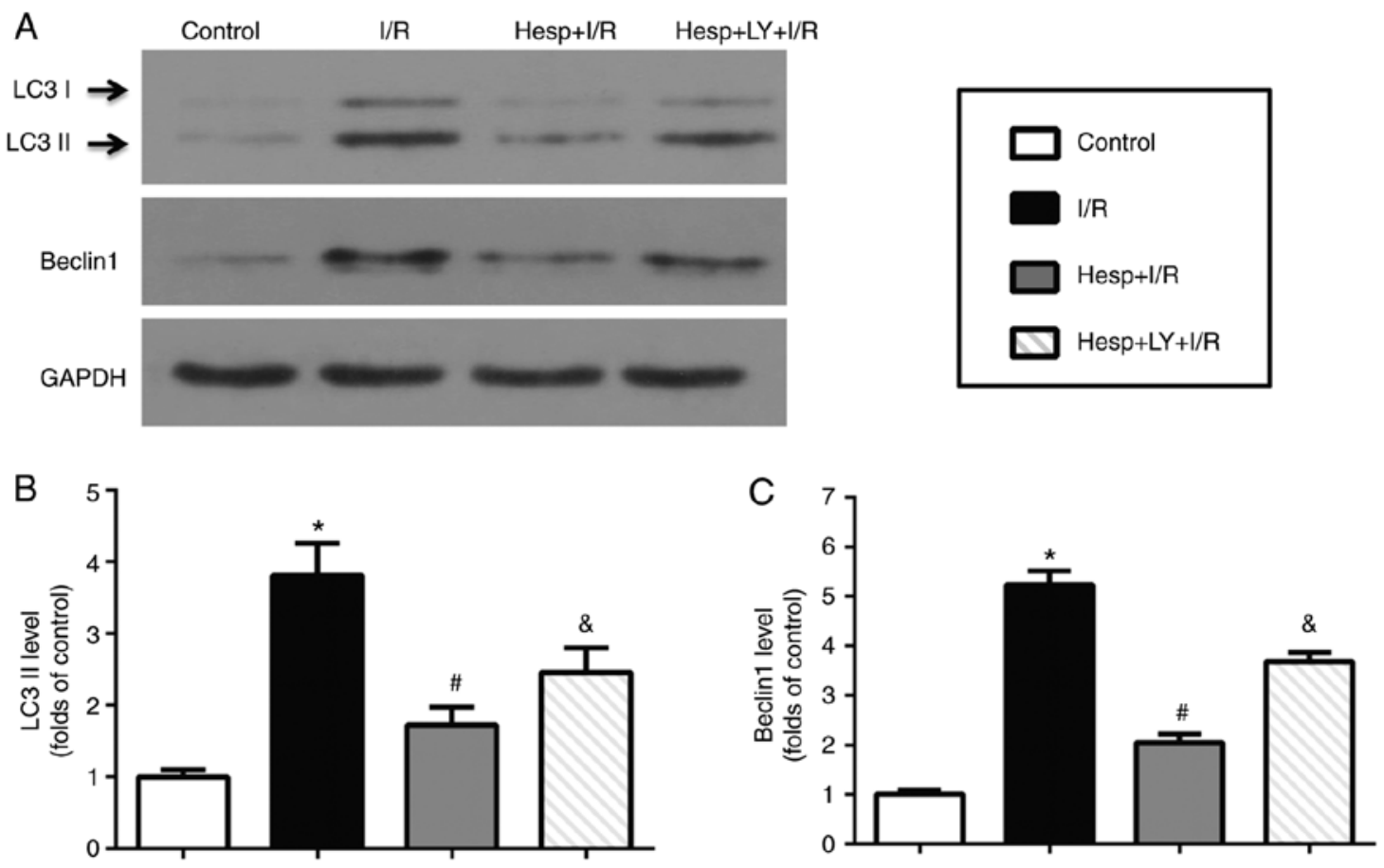

Figure 5. Effect of hesperidin on autophagy induced by I/R. (A) Representative examples of western blot analysis illustrating the expression levels of LC3II and Beclin1. (B) Expression level of LC3II ( $\mathrm{n}=5$ ). (C) Expression level of Beclin1 ( $\mathrm{n}=5)$. ${ }^{*} \mathrm{P}<0.05$ vs. Control group; ${ }^{*} \mathrm{P}<0.05$ vs. I/R group; ${ }^{\text {\& }} \mathrm{P}<0.05$ vs. Hesp $+\mathrm{I} / \mathrm{R}$ group. Hesp, hesperidin; I/R, ischemia/reperfusion; LY, LY294002; LC3 II, light chain 3 II. 

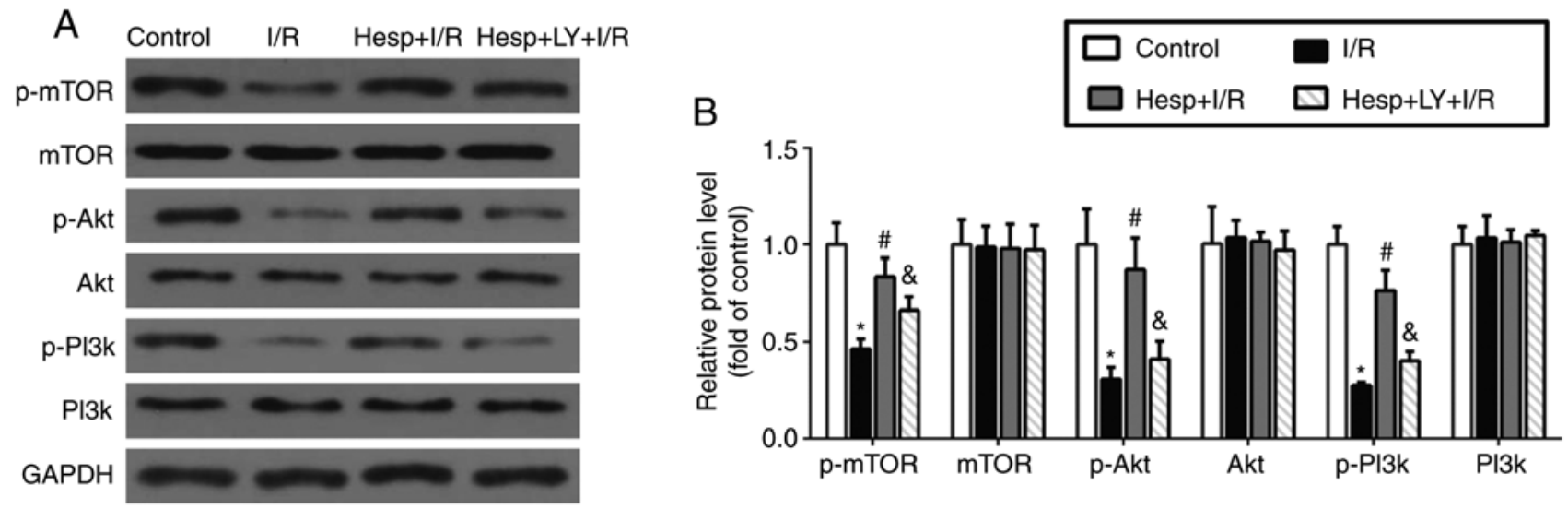

Figure 6. Effect of hesperidin on p-mTOR, mTOR, p-Akt, Akt, p-PI3K and PI3K expression levels in the myocardium during I/R. (A) Representative examples of western blot analysis demonstrating the expression levels of p-mTOR, mTOR, p-Akt, Akt, p-PI3K and PI3K. (B) Quantification of the expression levels of p-mTOR, mTOR, p-Akt, Akt, p-PI3K and PI3K ( $n=5)$. " $\mathrm{P}<0.05$ vs. Control group; ${ }^{*} \mathrm{P}<0.05$ vs. I/R group; ${ }^{\text {P }}<<0.05$ vs. the Hesp+I/R group. $\mathrm{p}-$, phosphorylated; mTOR, mammalian target of rapamycin; PI3K, phosphatidylinositol 3-kinase; Akt, protein kinase B; Hesp, hesperidin; I/R, ischemia/reperfusion; LY, LY294002.

\section{Discussion}

The present study aimed to investigate the potential mechanism of cardioprotection of hesperidin against ischemia/reperfusion (I/R), with a particular focus on autophagy. To the best of our knowledge, the present study was the first to demonstrate that hesperidin inhibited excessive autophagy following I/R, which was displayed by the downregulation of LC3II and Beclin1 expression levels. Furthermore, hesperidin was demonstrated to activate the PI3K/Akt/mTOR pathway, and LY294002, a specific PI3K inhibitor, significantly reversed the effects of hesperidin. These results suggested that inhibition of autophagy via activation of the $\mathrm{PI} 3 \mathrm{~K} / \mathrm{Akt} / \mathrm{mTOR}$ pathway contributes to the cardioprotective effect of hesperidin during $\mathrm{I} / \mathrm{R}$.

Irreversible loss of cardiomyocytes is the principal cause of myocardial injury induced by myocardial infarction or $\mathrm{I} / \mathrm{R}$, which leads to persistent cardiac dysfunction and heart failure (28). Necrosis, apoptosis, and autophagy, the three main mechanisms of cell death, are known to participate in the loss of cardiomyocytes induced by I/R (28). Of these, autophagy, an intracellular lysosomal degradative pathway, serves a key role in cellular homeostasis (7). LC3 and Beclin1 are important and reliable markers of autophagy. LC 3 exists in the cytosolic form (LC3I) and membrane bound form (LC3II) (29). Increased LC3II is considered to be closely associated with the extent of autophagosome formation (30). Beclin1 is essential in the recruitment of other autophagic proteins during expansion of the pre-autophagosomal membrane and structure (31).

Under basal conditions, autophagy functions as a cell survival mechanism and maintains the cell size, construction and function of cardiomyocytes (32). However, under the condition of $\mathrm{I} / \mathrm{R}$, autophagy is upregulated and its role in modulating cardiomyocyte survival and death remains controversial. Previous studies have reported that autophagy promotes cell survival by purging damaged proteins and organelles to generate the intracellular building blocks required to maintain vital functions during ischemia $(33,34)$; whereas other studies demonstrated that autophagy also promotes cell death via excessive self-digestion and degradation of essential cellular constituents $(9,34)$. In the present study, the immunofluorescence staining results demonstrated that the LC3 signals were markedly increased in the I/R group. The expression levels of LC3II and Beclin1 in the myocardium, myocardial infarct size and serum levels of CK-MB and cTnI were also significantly upregulated following reperfusion, indicating that autophagy is over-activated during $I / R$. These findings suggested that excessive autophagy contributes to the process of myocardial I/R injury.

A growing number of epidemiological studies have reported that diets rich in herbs, fruits and spices can attenuate the risk of cardiovascular diseases $(35,36)$. Hesperidin, a bioactive flavanone glycoside, is highly abundant in citrus fruit (12). In a randomized crossover study, Morand et al (37) reported that hesperidin (294 mg in $500 \mathrm{ml}$ of orange juice) contributes to the cardioprotective effect of orange juice in overweight men by decreasing diastolic blood pressure and increasing endothelium-dependent microvascular reactivity. Another double-blind, crossover study also reported that hesperidin treatment $(500 \mathrm{mg} / \mathrm{day})$ improves endothelial function and reduces inflammatory markers in patients with metabolic syndrome (38). Using the dose conversion of body surface area comparisons between humans and rats (1:6.17) (39), $200 \mathrm{mg} / \mathrm{kg} /$ day of hesperidin used in rats is equal to $1,944 \mathrm{mg} / \mathrm{day}$ for a $60-\mathrm{kg}$ man, which is 6-fold greater than $294 \mathrm{mg} / \mathrm{day}$ and 3 -fold greater than $500 \mathrm{mg} / \mathrm{day}$. Hence, the dose of hesperidin used in the present study far exceeded a nutritional dose; however, it functioned as a therapeutic dose.

The study of Huang et al (40) reported that hesperidin protects against amyloid $\beta$-induced neuronal injury through suppression of neuronal autophagy. In the present study, the data presented similar results, which revealed that hesperidin significantly downregulated the expression levels of LC3II and Beclin1 in the heart of rats, which was accompanied with low myocardial infarct size and low serum levels of CK-MB and cTnI. Combined, the aforementioned results indicated that excessive autophagy contributes to the process of myocardial $\mathrm{I} / \mathrm{R}$ injury, and the findings of the present study suggested that 
hesperidin could reduce myocardial I/R injury by inhibiting excessive autophagy.

Autophagy can be modulated by multiple signaling pathways, including the PI3K/Akt pathway (22). The PI3K/Akt pathway, a major regulator of cell growth and survival, serves an essential role in the mediation of myocardial cell survival in many situations (27). Previous studies demonstrated that activation of PI3K/Akt ameliorates myocardial I/R injury (20,24). Furthermore, activation of the PI3K/Akt pathway can phosphorylate mTOR, which is a key regulator of autophagy (22). Phosphorylated mTOR has been reported to protect against I/R injury by reducing autophagy and enhancing recovery in the heart (41). It has been reported that hesperidin activates the Akt pathway in a rat model of neuroinflammation induced by aluminum chloride (42). Saiprasad et al (43) also demonstrated that hesperidin regulates the PI3K/Akt/mTOR pathway in a mouse model of colon carcinogenesis. Similar results were obtained in the present study, which demonstrated that hesperidin significantly increased the expression levels of p-PI3K, p-Akt and p-mTOR and decreased the expression levels of LC3II and Beclin1, while LY294002, a specific inhibitor of PI3K, markedly reversed the aforementioned effects of hesperidin. These findings suggested that hesperidin inhibited excessive autophagy via activating the PI3K/Akt/mTOR pathway.

Although the present study suggested that hesperidin may be a novel drug for protection of myocardial I/R injury, there are still various limitations. Firstly, the dose of hesperidin used in the present study is a therapeutic dose; the effective range of hesperidin dose and related safety evaluation need to be investigated in further studies. Second, the effects of hesperidin on longer term myocardial remodeling following myocardial I/R injury also requires further exploration.

In conclusion, the present study demonstrated that hesperidin reduced myocardial I/R injury by suppressing excessive autophagy, and activation of the PI3K/Akt/mTOR pathway contributed to the inhibitory effect of hesperidin on excessive autophagy.

\section{Acknowledgements}

Not applicable.

\section{Funding}

No funding was received.

\section{Availability of data and materials}

The data used and/or analyzed during the present study are available from the corresponding author on reasonable request.

\section{Authors' contributions}

$\mathrm{XL}, \mathrm{XH}$, and $\mathrm{HJ}$ conceived and designed the experiment. XL, RM, CY and WX carried out the experiments. RM, CY and WX acquired the reagents and materials. XL and JW analyzed the data. XL, XH, JW and HJ wrote the manuscript. All authors approved the final version of the manuscript.

\section{Ethics approval and consent to participate}

The present study was approved by Institutional Animal Care and Use Committee of Wuhan University (approval no. 2016-0318).

\section{Patient consent for publication}

Not applicable.

\section{Competing interests}

The authors declare that they have no competing interests.

\section{References}

1. Roger VL, Go AS, Lloyd-Jones DM, Benjamin EJ, Berry JD, Borden WB, Bravata DM, Dai S, Ford ES, Fox CS, et al: Executive Summary: Heart disease and stroke statistics-2012 update: A report from the american heart association. Circulation 125: 188-197, 2012.

2. Yellon DM and Hausenloy DJ: Myocardial reperfusion injury. N Engl J Med 357: 1121-1135, 2007.

3. Ogura Y, Ouchi N, Ohashi K, Shibata R, Kataoka Y, Kambara T, Kito T, Maruyama S, Yuasa D, Matsuo K, et al: Therapeutic impact of follistatin-like 1 on myocardial ischemic injury in preclinical models. Circulation 126: 1728-1738, 2012.

4. Frangogiannis NG, Smith CW and Entman ML: The inflammatory response in myocardial infarction. Cardiovasc Res 53: 31-47, 2002.

5. Gottlieb RA and Engler RL: Apoptosis in myocardial ischemia-reperfusion. Ann N Y Acad Sci 874: 412-426, 1999

6. Przyklenk K, Dong Y, Undyala VV and Whittaker P: Autophagy as a therapeutic target for ischaemia/reperfusion injury? Concepts, controversies, and challenges. Cardiovasc Res 94: 197-205, 2012.

7. Marino G, Madeo F and Kroemer G: Autophagy for tissue homeostasis and neuroprotection. Curr Opin Cell Biol 23: 198-206, 2011.

8. Loos B, Engelbrecht AM, Lockshin RA, Klionsky DJ and Zakeri Z: The variability of autophagy and cell death susceptibility: Unanswered questions. Autophagy 9: 1270-1285, 2013.

9. Ke J, Yao B, Li T, Cui S and Ding H: A2 Adenosine receptor-mediated cardioprotection against reperfusion injury in rat hearts is associated with autophagy downregulation. J Cardiovase Pharmacol 66: 25-34, 2015.

10. Cao X, Chen A, Yang P, Song X, Liu Y, Li Z, Wang X, Wang L and Li Y: Alpha-lipoic acid protects cardiomyocytes against hypoxia/reoxygenation injury by inhibiting autophagy. Biochem Biophys Res Commun 441: 935-940, 2013.

11. Cheng BC, Huang HS, Chao CM, Hsu CC, Chen CY and Chang CP: Hypothermia may attenuate ischemia/reperfusion-induced cardiomyocyte death by reducing autophagy. Int J Cardiol 168: 2064-2069, 2013.

12. Garg A, Garg S, Zaneveld LJ and Singla AK: Chemistry and pharmacology of the Citrus bioflavonoid hesperidin. Phytother Res 15: 655-669, 2001.

13. Kuntic V, Brboric J, Holclajtner-Antunovic I and Uskokovic-Markovic S: Evaluating the bioactive effects of flavonoid hesperidin-a new literature data survey. Vojnosanit Pregl 71: 60-65, 2014.

14. Wei D, Ci X, Chu X, Wei M, Hua S and Deng X: Hesperidin suppresses ovalbumin-induced airway inflammation in a mouse allergic asthma model. Inflammation 35: 114-121, 2012.

15. Yumnam S, Hong GE, Raha S, Saralamma VV, Lee HJ, Lee WS, Kim EH and Kim GS: Mitochondrial dysfunction and $\mathrm{Ca}(2+)$ overload contributes to hesperidin induced paraptosis in hepatoblastoma cells, HepG2. J Cell Physiol 231: 1261-1268, 2016.

16. Petrova A, Davids LM, Rautenbach F and Marnewick JL: Photoprotection by honeybush extracts, hesperidin and mangiferin against UVB-induced skin damage in SKH-1 mice. J Photochem Photobiol B 103: 126-139, 2011. 
17. Kim SH, Kim BK and Lee YC: Antiasthmatic effects of hesperidin, a potential Th2 cytokine antagonist, in a mouse model of allergic asthma. Mediators Inflamm 2011: 485402, 2011.

18. Selvaraj P and Pugalendi KV: Hesperidin, a flavanone glycoside, on lipid peroxidation and antioxidant status in experimental myocardial ischemic rats. Redox Rep 15: 217-223, 2010.

19. Gandhi C, Upaganalawar A and Balaraman R: Protection against in vivo focal myocardial ischemia/reperfusion injury-induced arrhythmias and apoptosis by hesperidin. Free Radic Res 43 : 817-827, 2009

20. Li X, Hu X, Wang J, Xu W, Yi C, Ma R and Jiang H: Short-term hesperidin pretreatment attenuates rat myocardial ischemia/reperfusion injury by inhibiting high mobility group box 1 protein expression via the PI3K/Akt pathway. Cell Physiol Biochem 39: 1850-1862, 2016.

21. Wymann MP, Zvelebil M and Laffargue M: Phosphoinositide 3-kinase signalling-which way to target? Trends Pharmacol Sci 24: 366-376, 2003.

22. Shao X, Lai D, Zhang L and Xu H: Induction of autophagy and apoptosis via PI3K/AKT/TOR pathways by azadirachtin a in spodoptera litura cells. Sci Rep 6: 35482, 2016.

23. Rong Z, Pan R, Xu Y, Zhang C, Cao Y and Liu D: Hesperidin pretreatment protects hypoxia-ischemic brain injury in neonatal rat. Neuroscience 255: 292-299, 2013.

24. Wang J, Yang H, Hu X, Fu W, Xie J, Zhou X, Xu W and Jiang H: Dobutamine-mediated heme oxygenase-1 induction via PI3K and p38 MAPK inhibits high mobility group box 1 protein release and attenuates rat myocardial ischemia/reperfusion injury in vivo. J Surg Res 183: 509-516, 2013.

25. Ruisong M, Xiaorong H, Gangying H, Chunfeng Y, Changjiang Z, Xuefei L, Yuanhong L and Hong J: The protective role of interleukin-33 in myocardial ischemia and reperfusion is associated with decreased HMGB1 expression and up-regulation of the P38 MAPK signaling pathway. PloS One 10: e0143064, 2015.

26. Yang J, Jiang H, Yang J, Ding JW, Chen LH, Li S and Zhang XD: Valsartan preconditioning protects against myocardial ischemia-reperfusion injury through TLR4/NF-kappaB signaling pathway. Mol Cell Biochem 330: 39-46, 2009.

27. Wang ZG, Wang Y, Huang Y, Lu Q, Zheng L, Hu D, Feng WK, Liu YL, Ji KT, Zhang HY, et al: bFGF regulates autophagy and ubiquitinated protein accumulation induced by myocardial ischemia/reperfusion via the activation of the PI3K/Akt/mTOR pathway. Sci Rep 5: 9287, 2015.

28. Xu Q, Li X, Lu Y, Shen L, Zhang J, Cao S, Huang X, Bin J and Liao Y: Pharmacological modulation of autophagy to protect cardiomyocytes according to the time windows of ischaemia/reperfusion. Br J Pharmacol 172: 3072-3085, 2015.

29. Luo T, Liu G, Ma H, Lu B, Xu H, Wang Y, Wu J, Ge P and Liang J: Inhibition of autophagy via activation of PI3K/Akt pathway contributes to the protection of ginsenoside Rb1 against neuronal death caused by ischemic insults. Int J Mol Sci 15: 15426-15442, 2014.

30. Kabeya Y, Mizushima N, Ueno T, Yamamoto A, Kirisako T, Noda T, Kominami E, Ohsumi Y and Yoshimori T: LC3, a mammalian homologue of yeast Apg8p, is localized in autophagosome membranes after processing. EMBO J 19: 5720-5728, 2000 .
31. Liang XH, Jackson S, Seaman M, Brown K, Kempkes B, Hibshoosh $\mathrm{H}$ and Levine B: Induction of autophagy and inhibition of tumorigenesis by beclin 1. Nature 402: 672-676, 1999.

32. Godar RJ, Ma X, Liu H, Murphy JT, Weinheimer CJ, Kovacs A, Crosby SD, Saftig P and Diwan A: Repetitive stimulation of autophagy-lysosome machinery by intermittent fasting preconditions the myocardium to ischemia-reperfusion injury. Autophagy 11: 1537-1560, 2015.

33. Kuma A, Hatano M, Matsui M, Yamamoto A, Nakaya H, Yoshimori T, Ohsumi Y, Tokuhisa T and Mizushima N: The role of autophagy during the early neonatal starvation period. Nature 432: 1032-1036, 2004.

34. Hamacher-Brady A, Brady NR, Logue SE, Sayen MR, Jinno M, Kirshenbaum LA, Gottlieb RA and Gustafsson AB: Response to myocardial ischemia/reperfusion injury involves Bnip3 and autophagy. Cell Death Differ 14: 146-157, 2007.

35. Mink PJ, Scrafford CG, Barraj LM, Harnack L, Hong CP, Nettleton JA and Jacobs DR Jr: Flavonoid intake and cardiovascular disease mortality: A prospective study in postmenopausal women. Am J Clin Nutr 85: 895-909, 2007.

36. Banerjee SK and Maulik SK: Effect of garlic on cardiovascular disorders: A review. Nutr J 1: 4, 2002.

37. Morand C, Dubray C, Milenkovic D, Lioger D, Martin JF, Scalbert A and Mazur A: Hesperidin contributes to the vascular protective effects of orange juice: A randomized crossover study in healthy volunteers. Am J Clin Nutr 93: 73-80, 2011.

38. Rizza S, Muniyappa R, Iantorno M, Kim JA, Chen H, Pullikotil P, Senese N, Tesauro M, Lauro D, Cardillo C and Quon MJ: Citrus polyphenol hesperidin stimulates production of nitric oxide in endothelial cells while improving endothelial function and reducing inflammatory markers in patients with metabolic syndrome. J Clin Endocrinol Metab 96: E782-E792, 2011

39. Reagan-Shaw S, Nihal M and Ahmad N: Dose translation from animal to human studies revisited. FASEB J 22: 659-661, 2008.

40. Huang SM, Tsai SY, Lin JA, Wu CH and Yen GC: Cytoprotective effects of hesperetin and hesperidin against amyloid $\beta$-induced impairment of glucose transport through downregulation of neuronal autophagy. Mol Nutr Food Res 56: 601-609, 2012.

41. Sciarretta S, Volpe M and Sadoshima J: Mammalian target of rapamycin signaling in cardiac physiology and disease. Circ Res 114: 549-564, 2014.

42. Justin-Thenmozhi A, Dhivya Bharathi M, Kiruthika R, Manivasagam T, Borah A and Essa MM: Attenuation of aluminum chloride-induced neuroinflammation and caspase activation through the AKT/GSK-3 $\beta$ pathway by hesperidin in wistar rats. Neurotox Res, 2018.

43. Saiprasad G, Chitra P, Manikandan R and Sudhandiran G: Hesperidin induces apoptosis and triggers autophagic markers through inhibition of aurora-A mediated phosphoinositide-3-kinase/Akt/mammalian target of rapamycin and glycogen synthase kinase-3 beta signalling cascades in experimental colon carcinogenesis. Eur J Cancer 50: 2489-2507, 2014.

This work is licensed under a Creative Commons Attribution-NonCommercial-NoDerivatives 4.0 International (CC BY-NC-ND 4.0) License. 